\title{
Ordered One-Component Plasmas: Phase Transitions, Normal Modes, Large Systems, and Experiments in a Storage Ring
}

\author{
J. P. Schiffer \\ Physics Division, Argonne National Laboratory, Argonne, IL 60439 \\ and The University of Chicago, Chicago, IL 60637
}

The property of cold one-component plasmas, confined by external forces, to form an ordered array has been known for some time both from simulations (1) and from experiment (2). The purpose of this talk is to summarize some recent work on simulations and some new experimental results.

As a reminder of the qualitative features of this ordering, I would like to show the result of, what I believe, is the largest number of ions followed in a simulation. This is a system of 20,000 ions in isotropic confinement with a constant harmonic force, that was allowed to find its lowest potential energy in a Molecular Dynamics simulation -- whose essential features have been described before. This calculation was carried out over a period of almost a year by shepherding it through the NERSC Crays at low priority. The simulation requires computing the forces between all particles: $1 / 2 \times(20,000)^{2}$ calculations at each time step for many thousands of time steps. The total computer time invested represents on the order of 1000 actual Cray cpu hours. The motivation behind this calculation was, in part, to try and understand how far the layered structures seen in the simulation of smaller systems (1) continued in larger ones. After all, one knows that in an infinite system a bcc configuration has the lowest potential energy. Dubin (3), had estimated that possibly bcc order may win out in the interior after about 20 layers -- but this estimate did not include the potential energy in forming an interface between the two forms of ordering.

The type of order seen is shown in figure 1. The upper part of the figure shows the ordering of ions on the outer shell of the cold 20,000 ion system, with the triangular pattern that is characteristic of two-dimensional Coulombic systems. The lower part of the figure shows the radial density and indicates that the shell structure continues all the way to the interior, forming 18 distinct shells. The ordering of particles in each shell is similar to that in the outermost one -- though the thickness of the shells is somewhat greater. Table 1 indicates the data on all the shells seen in this simulation -- and several features are evident:

a) that the shells are equally spaced,

b) that the number of particles in each shell is proportional to the shell's radius (in other words that the surface density in each shell is constant, except that the outermost shell has about $1 \%$ higher surface density than all the others,

c) that the thickness of the shells increases gradually inward.

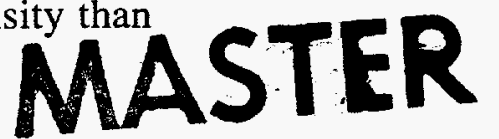
under contract No. W-31-109ENG-38. Accordingly, the U.S. Government retains a nonexclusive, royalty-free license to publish or reproduce the published form of this U. S. Government purposes. 


\section{DISCLAIMER}

Portions of this document may be illegible in electronic image products. Images are produced from the best available original document. 


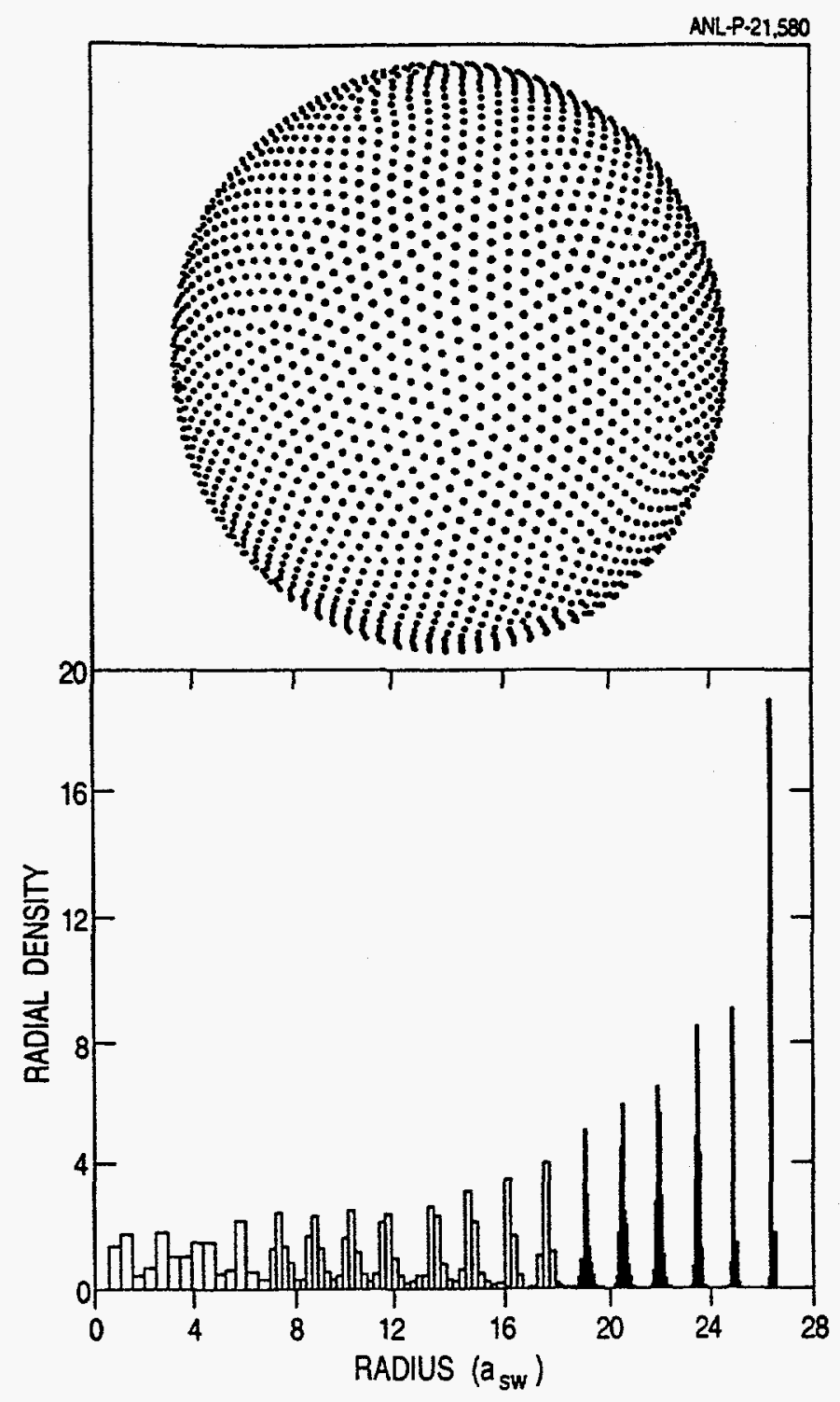

FIGURE 1. Simulation of 20,000 ions in an isotropic trap. On top, the outer shell of ions is shown, on the bottom the radial density. The distance scale is in units of the SeitzWigner radius $\left(4 / 3 \pi\right.$ as $\left.w^{3}=1 / \rho\right)$.

The simulation reached a minimum in the potential energy which corresponds to the configuration presented -- but it is not clear whether this minimum is the true minimum or whether it is a local one. Thus one cannot really speak of a value of the coupling parameter $\Gamma$ to characterize the system -- in a sense $\Gamma$ is infinite within the accuracy of the simulation - the classical temperature is zero. It would seem that this structure therefore is still stable for the 18-shell structure seen here. In the future one might try such a simulation with a bcc seed cell in the interior to see whether a lower potential energy might be reached. 
TABLE 1. Shell Structure in the 20,000 lon System ${ }^{a}$

\begin{tabular}{cccccc}
\hline $\begin{array}{c}\text { Shell } \\
\text { Number }\end{array}$ & $\begin{array}{c}\text { Shell } \\
\text { Radius }\end{array}$ & $\begin{array}{c}\text { Shell } \\
\text { Spacing }\end{array}$ & $\begin{array}{c}\text { Shell } \\
\text { Width }\end{array}$ & $\begin{array}{c}\text { Number } \\
\text { in Shell }\end{array}$ & $\begin{array}{c}\text { Excess } \\
\text { b }\end{array}$ \\
\hline 1 & 26.399 & 1.4753 & .0195 & 3116 & 38 \\
2 & 24.924 & 1.4701 & .0547 & 2743 & 0 \\
3 & 23.454 & 1.4707 & .0848 & 2423 & -6 \\
4 & 21.983 & 1.4695 & .107 & 2138 & 4 \\
5 & 20.514 & 1.4652 & .125 & 1847 & -11 \\
6 & 19.049 & 1.4705 & .148 & 1599 & -3 \\
7 & 17.578 & 1.4683 & .167 & 1366 & 2 \\
8 & 16.110 & 1.4602 & .184 & 1138 & -8 \\
9 & 14.650 & 1.4694 & .206 & 940 & -8 \\
10 & 13.180 & 1.4732 & .243 & 773 & 6 \\
11 & 11.707 & 1.4693 & .244 & 602 & -3 \\
12 & 10.238 & 1.4683 & .270 & 464 & 1 \\
13 & 8.770 & 1.4686 & .274 & 338 & -2 \\
14 & 7.301 & 1.443 & .285 & 235 & 0 \\
15 & 5.858 & 1.4178 & .253 & 144 & -8 \\
16 & 4.440 & 1.4787 & .289 & 86 & -1 \\
17 & 2.962 & 1.4754 & .294 & 39 & 0 \\
18 & 1.486 & & .209 & 9 & -1
\end{tabular}

aAll distances are in units of the Seitz-Wigner radius. "The "excess" is the number in a shell over (or under) what one would expect if the surface density in each shell were a constant.

The correlations between particles in these systems may be studied. For instance, the correlation between particles in one shell shows very pronounced structure that is characteristic of this two-dimensional order, but the correlation between particles in different shells does not show pronounced peaks. Figure 2a and $2 \mathrm{~b}$ show the correlation functions $\mathrm{g}(\mathrm{r})$ for a single shell, and for the whole system. The correlation as a function of the coordination, the total number of particles within a given distance from an initial particle, is also shown in figure 3.

While there is no obvious longer-range correlation between particles in different shells, other than that between nearest neighbors and successive shell layers, careful inspection of the adjacent layers indicates that the orientation of the two-dimensional lattices does tend to be similar from shell to shell. Figure 4 shows the correlation between the angular orientation of the lattices as a function of shell separation; note that the structure is symmetric for 60 degree rotations and so an uncorrelated system should yield 15 degrees for the value of this correlation (since 30 degrees is equivalent to - 30 degrees, and an uncorrelated system would imply the mean between 0 and 30). The figure indicates that the value of correlation function between adjacent shells is 4 degrees, and increases to 15 degrees in 8 shells. So clearly there is a correlation in the orientation of these systems that persists for many layers, even when the radial correlation function $\mathrm{g}(\mathrm{r})$ does not reflect it. 


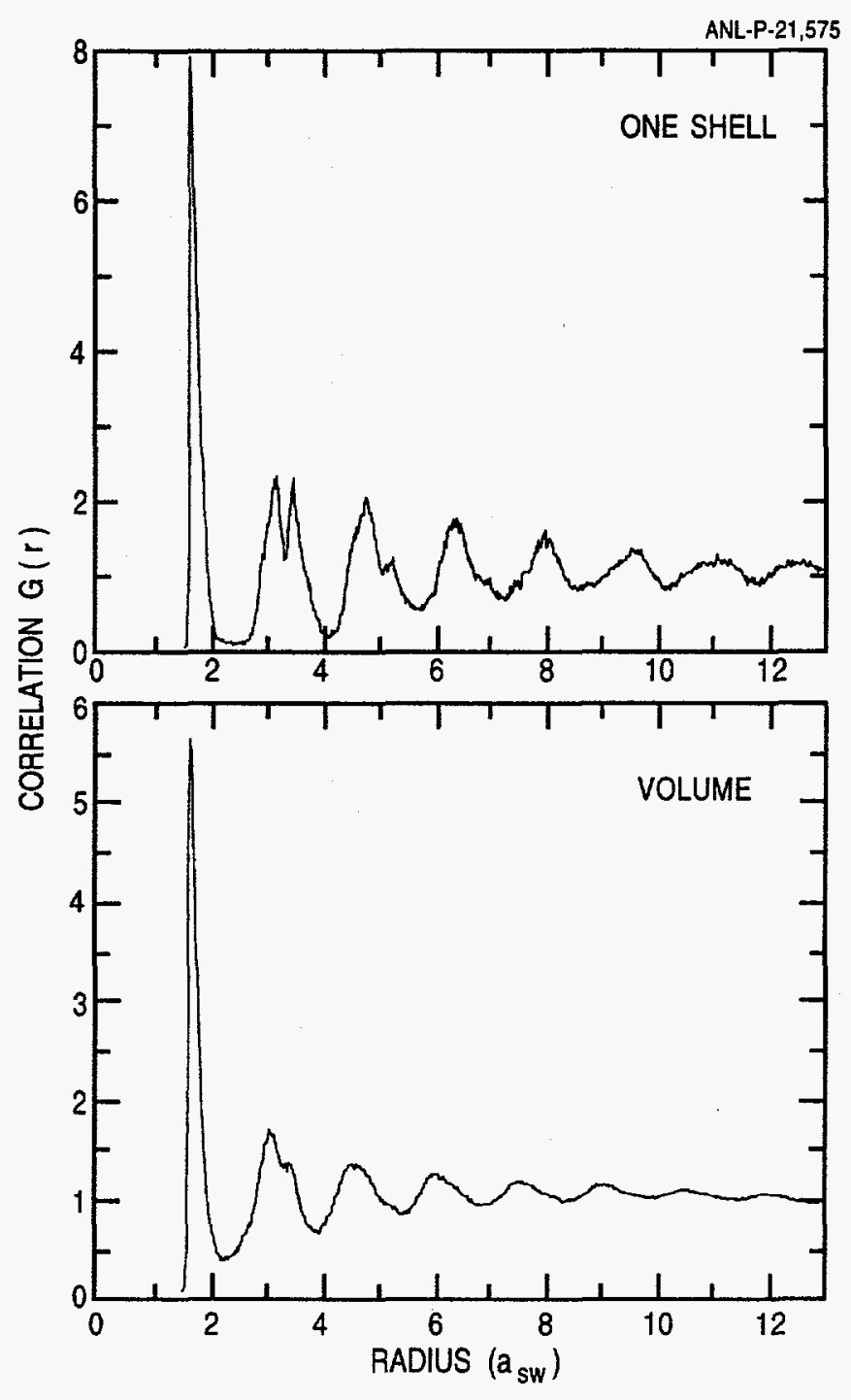

FIGURE 2. Correlation function in the spacing of ions. The top plot is the correlation function restricted to one shell -- on the bottom that for the whole volume -- but excluding the ions in the outer shells, in order to avoid surface effects.

This set of coordinates for the 20,000 particles under isotropic confinement could be the test bed for other calculations and the numbers are available on request.

Next I would like to show some simulations of the same number of particles under anisotropic confinement -- changing the confining force in the $\mathrm{z}$-direction by a factor of 5 . These simulations were not followed quite to a local minimum though $\Gamma$ is at least on the order of $10^{4}$-- an average kinetic energy is difficult to estimate when the system is being cooled, because generally one does not wait for the system to become completely equilibrated before lowering the temperature (it may take several thousand time steps to establish some sort of equilibrium value 


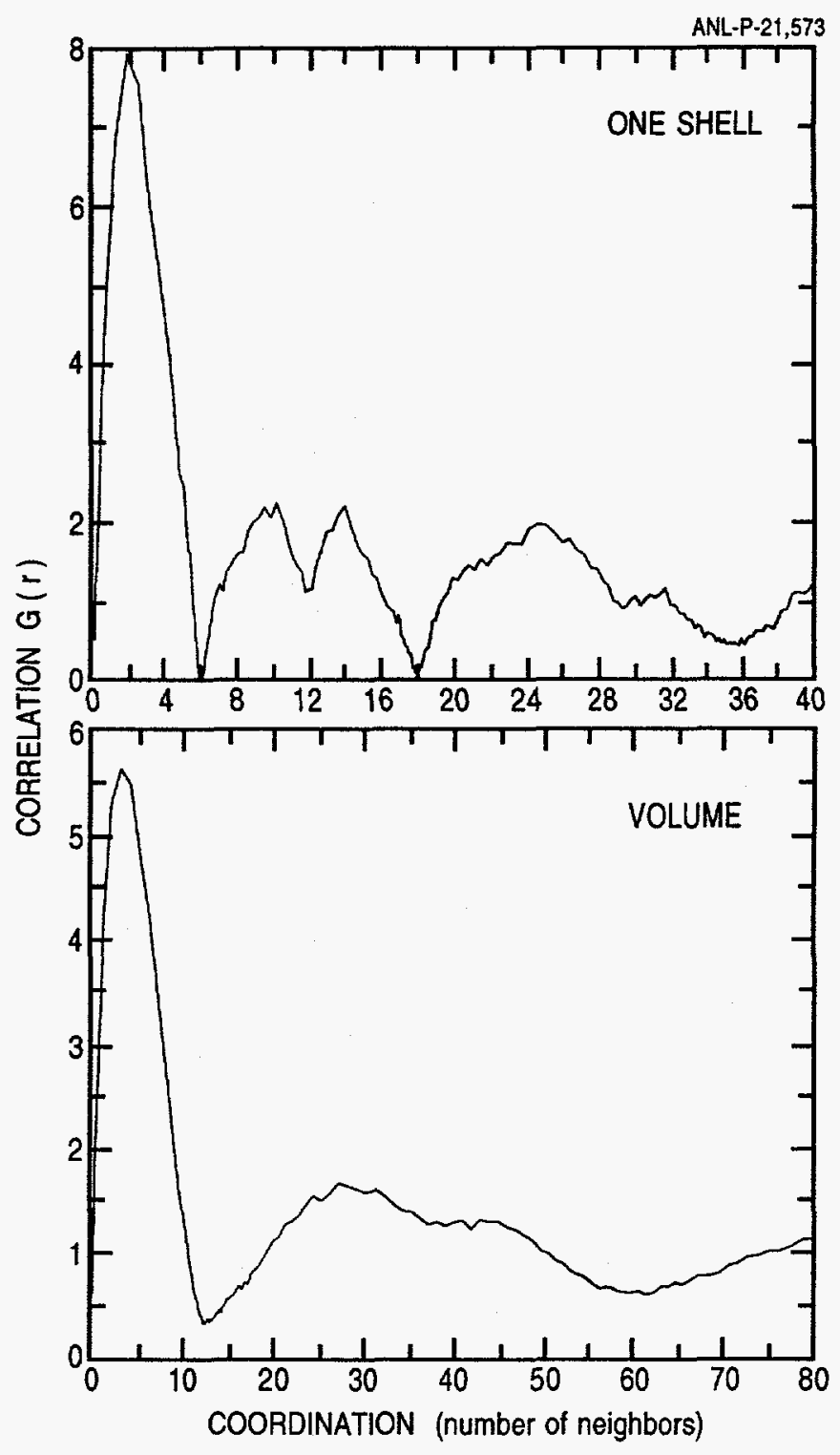

FIGURE 3. The same correlation function as in figure 2 , but plotted against the integral of nearest neighbors. For the single shell the first peak corresponds to a total of 6 nearest neighbors. For the volume correlation it is about 14.

for the kinetic energy). The results for these anisotropically confined systems are shown in figure 5. What one might expect naively is that the ion cloud would take on a spheroidal shape along equipotentials and that the interior layers would form additional spheroids. However, this is not the case, as may be seen by a closer examination of the data in figure 5. The outer shell of the system is indeed spheroidal. But the interior shells are not spheroids. One might have expected that the interior shells would be more deformed spheroids than the outer one, since if the shells are equally spaced (as in the spherical case), an equal step in the major and minor axes would lead to increasing eccentricities in these shells. 


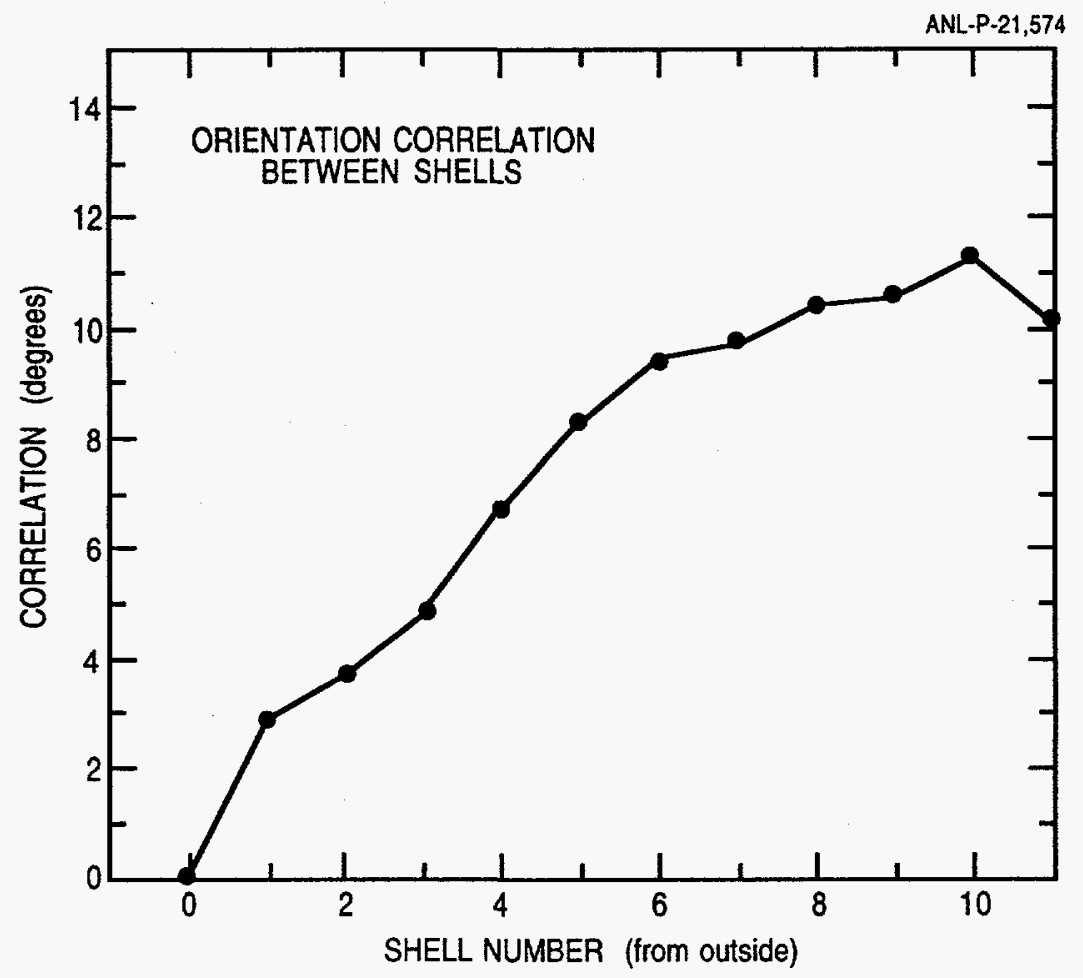

FIGURE 4. Correlation in the directional orientation of the two-dimensional structures in successive shells. Random orientations would yield 15 degrees -- so some correlation persists for 10 or more shells.

Instead of spheroids the particles seem to form shells that are at all points equidistant from the outermost shells by a constant perpendicular distance. This indeed leads to the formation of interior shells of increasing eccentricity but they are not spheroidal, but develop sharp points at the narrowest end. This feature of the calculations was certainly not expected a priori.

Next, I would like to review another aspect of asymmetric confinement that was found in simulations, that of dimensional phase transitions (4). Consider a system of few ions confined in a trap -- say 4 as is illustrated in figure 6 . If the trap parameters are similar to the "linear trap" configuration (5), with the confining force in the $z$-direction much weaker than in the radial direction, then the 4 ions will form a one-dimensional array and all sit on the z-axis. As the force in the zdirection is increased the ions will first pop out to form a rhomboid in 2 dimensions with all four simultaneously leaving the $z$ axis at $F_{z}>0.747$, then form a rhombus with two ions on the $\mathrm{z}$ axis, and two ions at $\mathrm{z}=0$ away from the axis at $F_{z}=0.872$, then the ions form a three-dimensional tetrahedron with one edge parallel to the $\mathrm{z}$ axis and one perpendicular starting at $\mathrm{F}_{\mathrm{z}}=0.912$, the tetrahedron becomes a regular tetrahedron with no preferred orientation but only for $F_{z}=1$, then the ions will form a tetrahedron with two edges parallel to the $z$ axis, and finally collapse into a two-dimensional square for $F_{z}>1.47$. Thus even this simple 4-ion system will have undergone 6 shape transitions as the trap parameters (the anisotropy in the confining force) are changed continuously, three of these are changes in the dimensionality of the system. These dimensional 


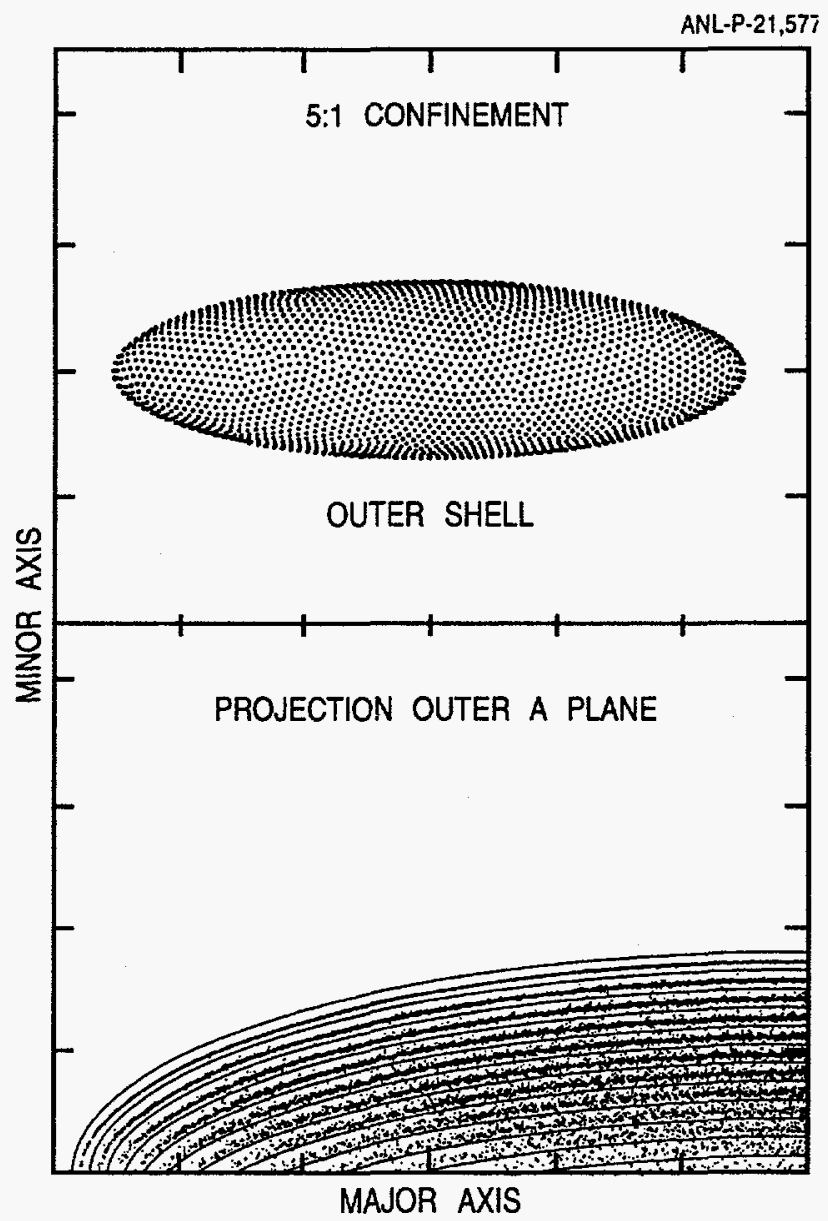

FIGURE 5. Simulation of 20,000 ions in anisotropic confinement with the restoring force in the $z$-direction 5 times less than in $x$ and $y$. The ions in the outer spheroidal shell are shown on top, while on the bottom a projection of particles onto the z-p plane is plotted. The lines are drawn to emphasize the fact that in the interior the ions do not settle onto spheroidal surfaces, but on ones that are a fixed perpendicular distance from the outer (spheroidal) shell.

changes are a general feature of any number of ions; the aspect ratio of a trap where the configuration changes from 1 to 2 , from 2 to 3 , and from 3 back to 2 dimensions, changes smoothly with the number of ions. This behavior was found in simulations and very shortly thereafter explained in terms of simple hydrodynamic considerations by Dubin (6).

Next I would like to show some results on normal modes of spherical clouds. This is an ongoing collaboration with Dan Dubin where we are trying to understand the effect of the correlations (of the crystalline structure) on the hydrodynamic normal modes that are apparent in these systems. Some results are shown in figure 7. It is evident, for instance, that the simplest (monopole) mode, which is a shape-conserving volume oscillation of a spherical cloud, shows no damping. On the other hand, a quadruple mode, which is a shape oscillation, shows appreciable damping -- after several hundred oscillations. We are trying to 


\section{FOUR PARTICLES WITH CHANGING FORCE IN 2}
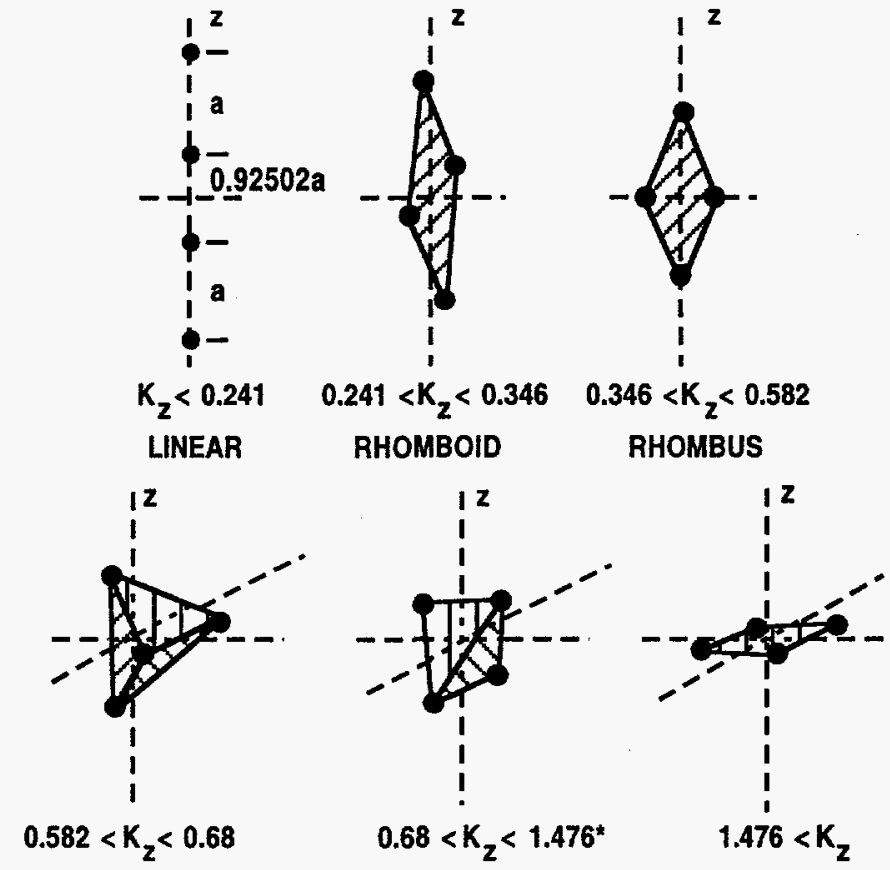

TETRAHEDRON ONE EDGE $\perp$ TO $z$

TETRAHEDRON TWO EDGES $\perp$ TO $z$

ONE !! * SINGULAR POINT AT $K_{z}=1.000$

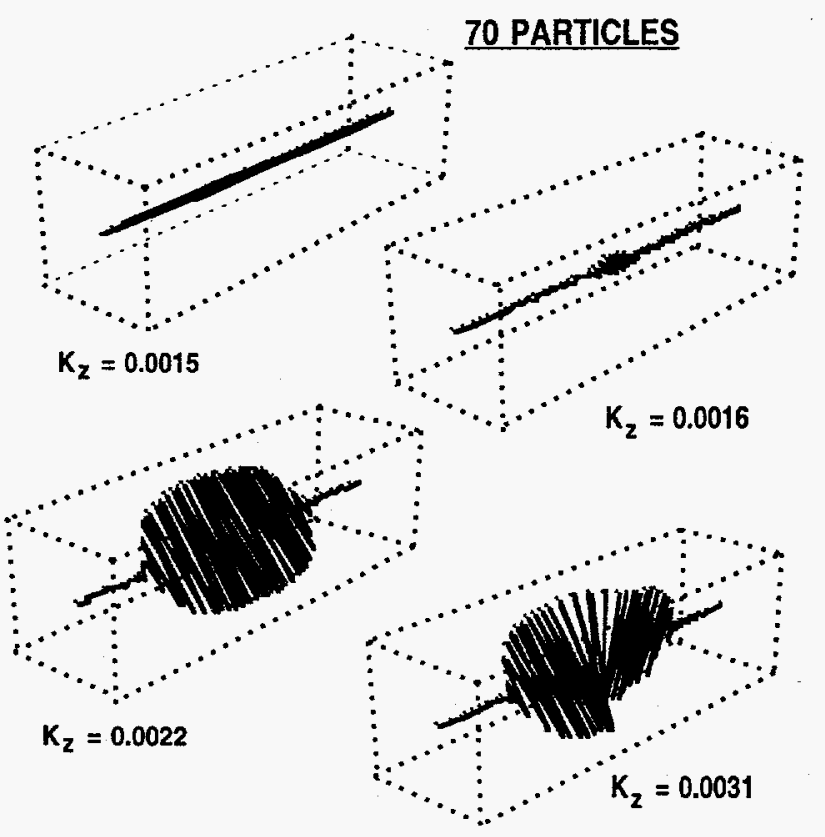

FIGURE 6. Illustration of dimensional phase transitions in the simulation of ions under anisotropic confinement. On top six distinct classes of shapes are shown as the focusing in the $z$-direction is changed (relative to the focusing in $x$ and $y$ ). On the bottom, some of the changes in the configurations for 70 ions are shown. Lines are drawn to connect the ions to emphasize the shapes of the configurations, and on the bottom curves the transverse scale is enlarged by a factor of 10 . 


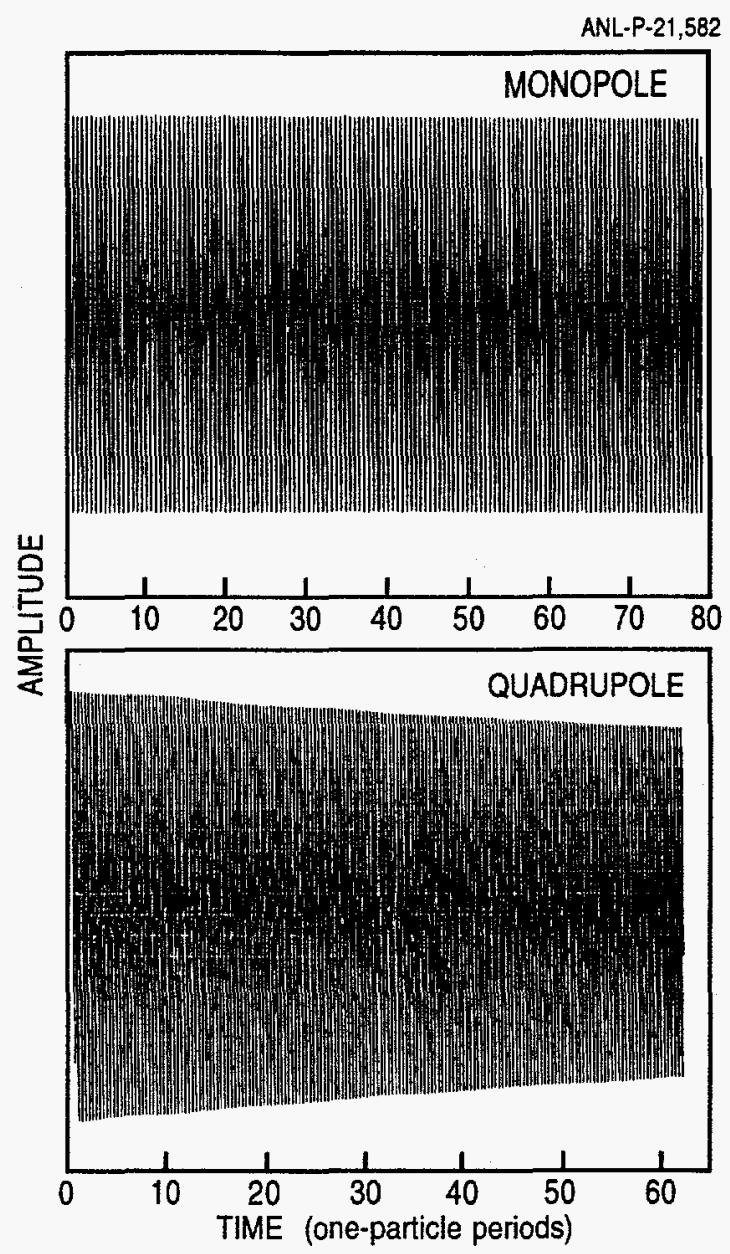

FIGURE 7. Oscillations of a cold ion cloud of 1000 ions in isotropic confinement, when subjected to a perturbation the excites a pure radial mode (top) and when a quadrupole mode is excited. Time is in units of the period of one particle oscillating in the same confining field.

understand this damping, and small deviations in the frequencies of these modes, more quantitatively. Then there are modes that are only possible in a crystalline system -- torsional modes may exist only from the interparticle interactions in an ordered system -- since a liquid has no resistance to shear. An example of this is given in figure 8.

I would now like to turn to some experimental work on real storage rings, magnetic storage devices in which particles circulate with large kinetic energies and for which laser cooling is used on partially ionized ions to attain temperatures ten or more orders of magnitude lower than their uniform kinetic energies. I cannot review the subject here and only want to mention two recent experiments at the ASTRID storage ring in Aarhus. In one of these, a single laser was used to cool a beam that was bunched by an rf system in the ring. This geometry is essentially that of a linear trap discussed earlier, with an aspect ratio on the order of 1000 or more. Without laser cooling, particles that are bunched undergo 


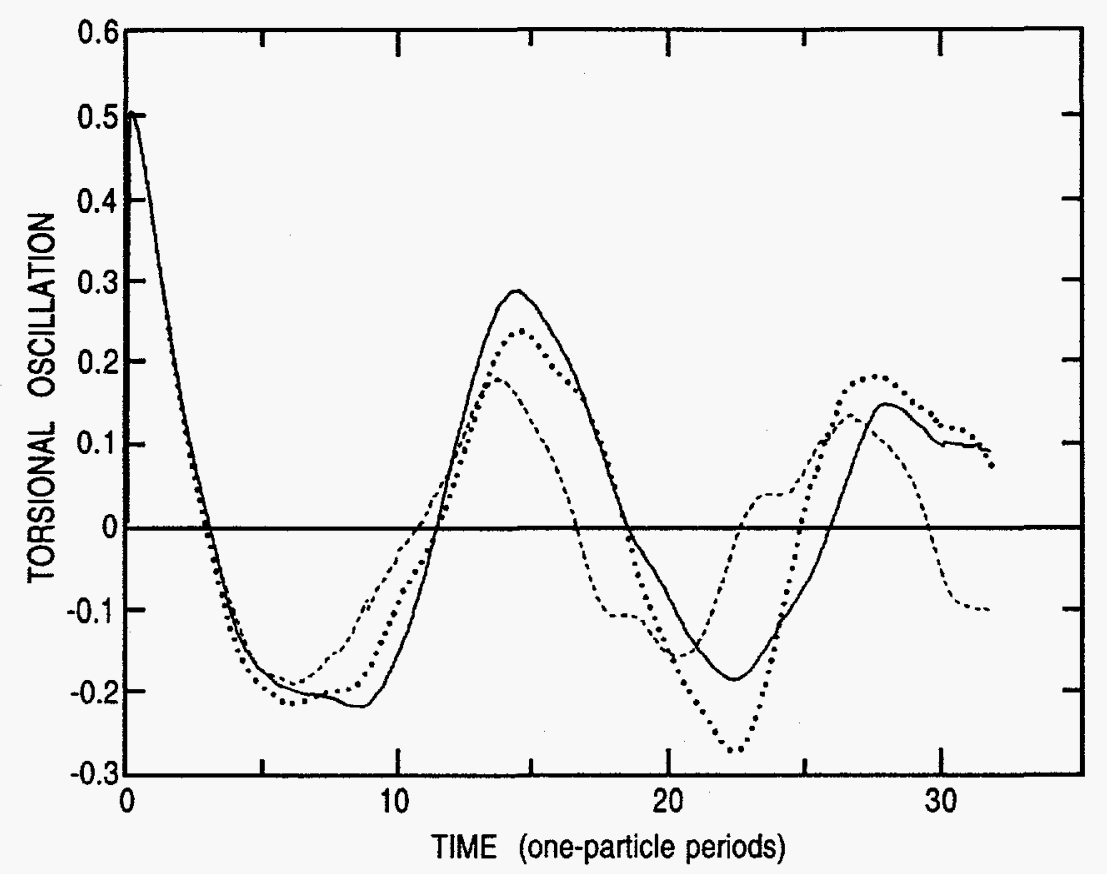

FIGURE 8. Torsional oscillations of a cold 1000 ion cloud. The three curves correspond to the response to the same perturbation applied about the $x, y$, and $z$ axes, they are slightly different because the finite cloud is not exactly symmetric, yet the oscillations are similar.

longitudinal oscillation in the rf fields, with a period that is large compared to the circulation period in the ring (the so-called synchrotron oscillations). When laser cooling is applied from one direction, these oscillations are damped out and the particles should be confined to a much tighter bunch in the ring. This has now been observed experimentally at ASTRID as shown in figure 9.

Another line of investigation is to use two lasers and cool a continuous ion beam between the two cooling forces. This we have also attempted recently and found to work. In figure 10 are shown two sets of signals -- one is the velocity spread of the ions as the laser cooling progresses, and the other is the 'Schottky signal', which is the Fourier transform of the noise signal from irregularities in the particle distribution. The splitting in the Schottky signal is from longitudinal wave propagation along the beam. Note that as the beam gets colder the logarithmic Schottky signal decreases, as it should. These results are both very new -- and indicate substantial progress in cooling these moving plasmas -however we have still quite a way to go before talking of crystallization in these systems.

I hope this report of various aspects of the field gives you a flavor of the many fascinating aspects of such condensed systems.

This research was supported by the U.S. Department of Energy, Nuclear Physics Division, under Contract W-31-109-Eng-38. The calculations reported were done on the NERSC CRAYs. 


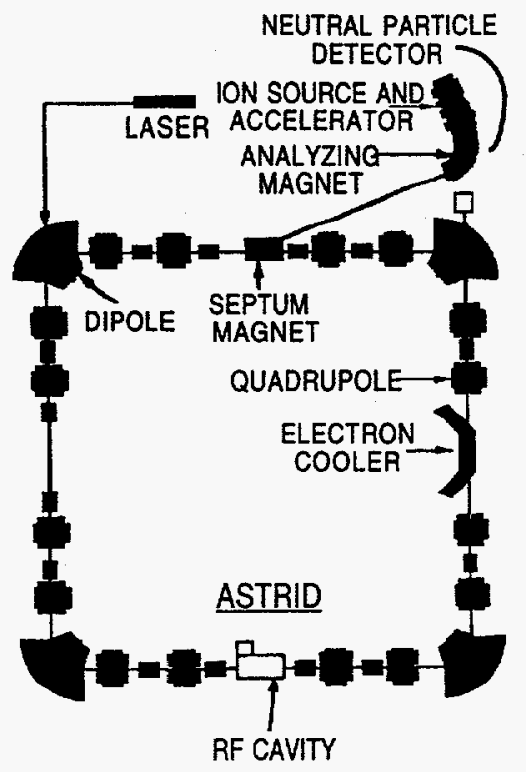

(a)

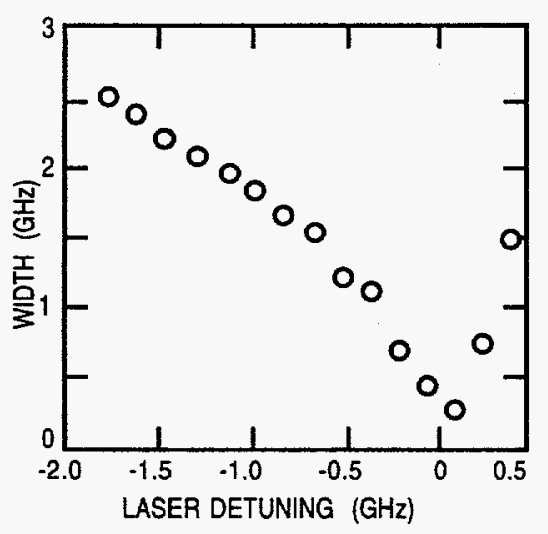

(c)

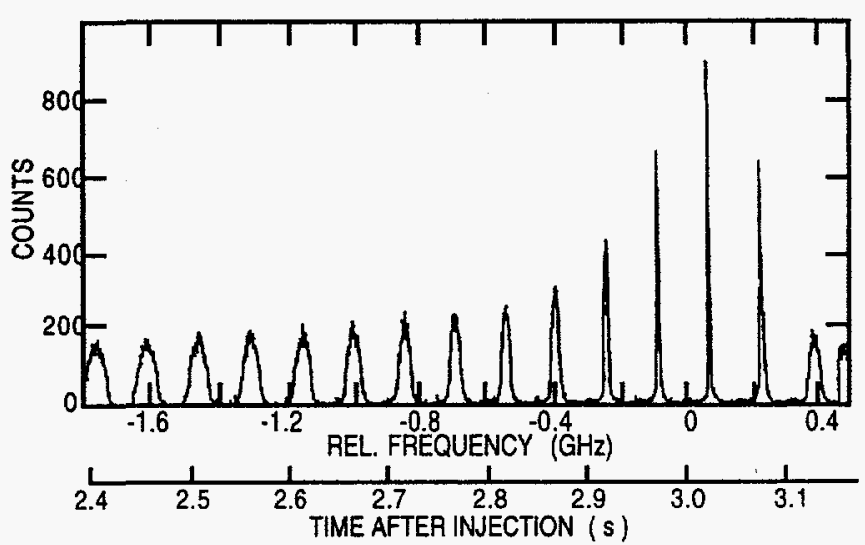

(b)

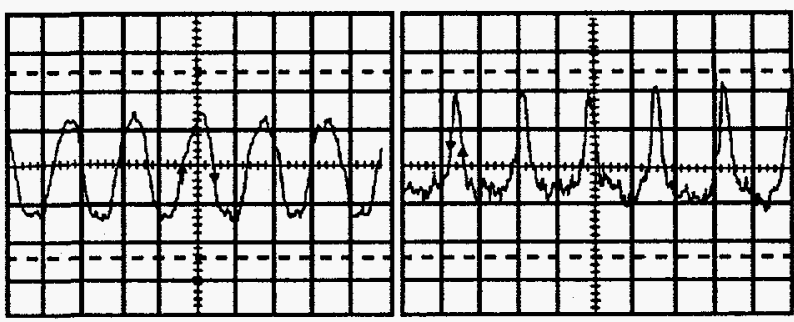

(d)

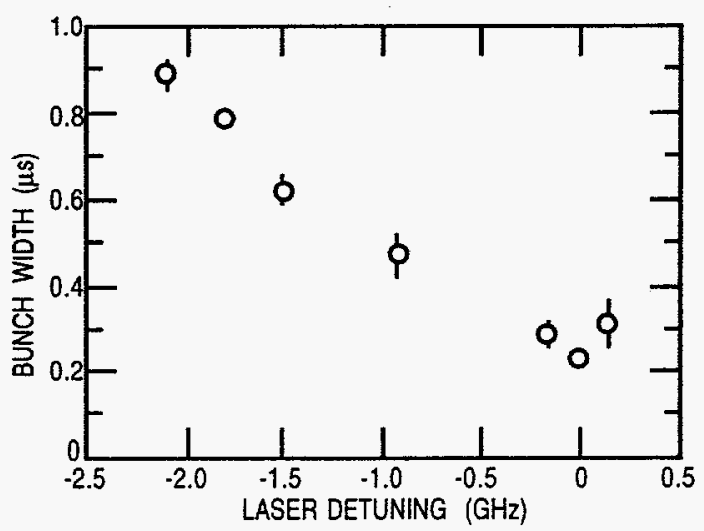

(e)

FIGURE 9. Results from laser cooling $\mathrm{Mg}^{+}$ions in a storage ring bunched into 23 longitudinal bunches while traveling around the ring with kinetic energies of $100 \mathrm{keV}$, obtained with J. S. Hangst, J. S. Nielsen, O. Poulsen, P. Shi, and B. Wanner. The ASTRID storage ring in Aarhus is shown schematically in (a). The laser scans with a probe laser of the beam are shown in (b), showing the sharpening velocity distribution of the ions as the laser cooling progresses; (c) shows the widths extracted from these scans. In (d) the physical length of the bunched beams is shown at two stages in laser cooling from the signal on pickup electrodes, and in (e) the bunch widths (lengths) extracted from these data are shown. 


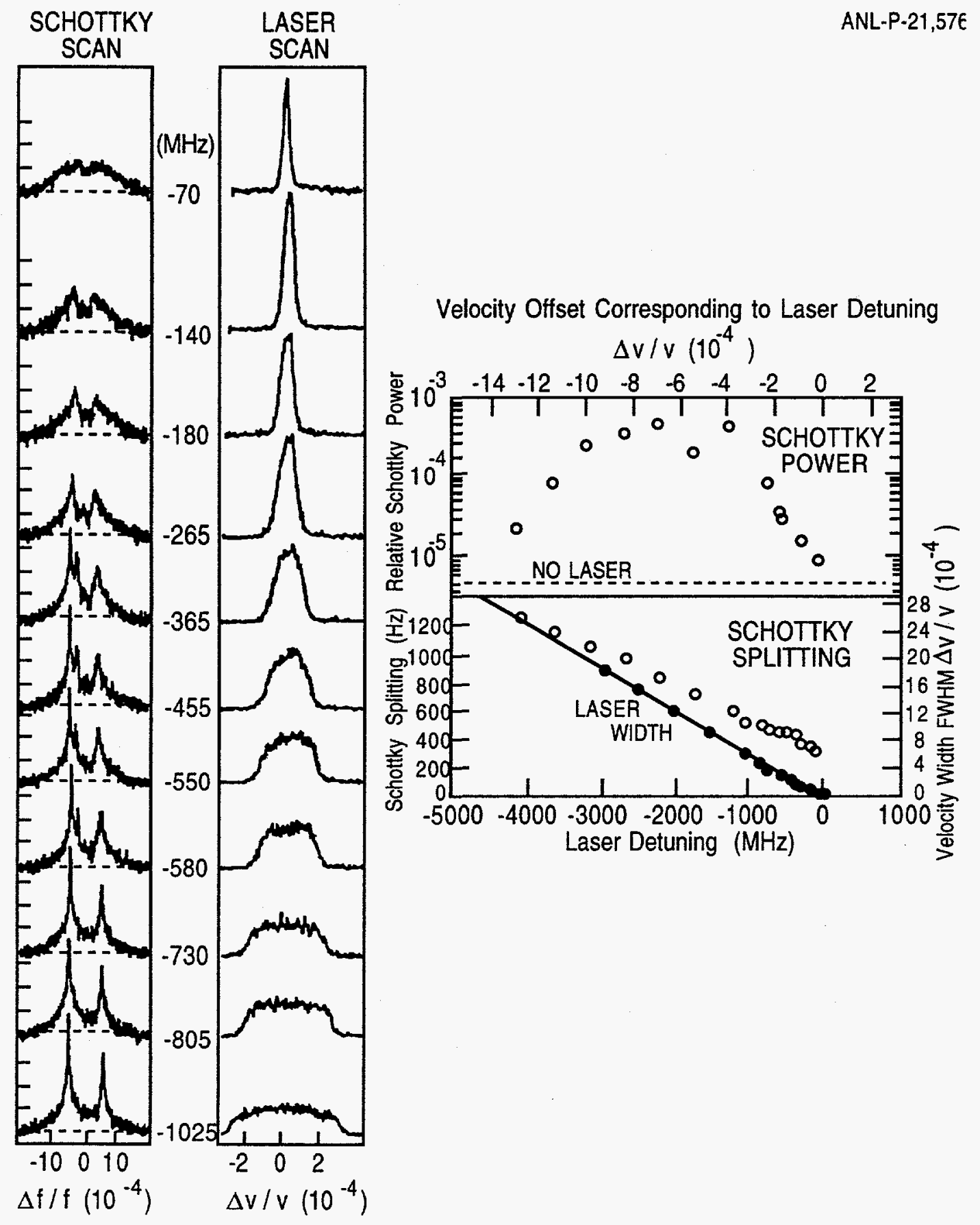

FIGURE 10. Results of laser cooling a continuous beam of $\mathrm{Mg}$ ions in the ASTRID storage ring (with J. S. Hangst, A. Labrador, V. Lebedev, N. Madsen, J. S. Nielsen, O. Poulsen, and $P$. Shi). On the left the velocity scans from a probe laser are shown as a function of laser cooling, along with the Schottky signal from pickup electrodes. On the right the total power in the Schottky signal, and the widths extracted from the data on the left are plotted. 


\section{REFERENCES}

1. Rahman, A. and Schiffer, J. P., Phys. Rev. Lett. 57, 1133-1136 (1986); Dubin, D. H. E. and O'Neal, T. M., Phys. Rev. Lett. 56, 728-731 (1986).

2. Gilbert, S. L., Bollinger, J. J., and Wineland, D. J., Phys. Rev. Lett. 60, 2022-2025 (1988);

Birkl, G., Kassner, S., and Walther, H., Nature 357, 310-313 (1992).

3. Dubin, D. H. E., Phys. Rev, A 40, 1140-1143 (1989).

4. Schiffer, J. P., Phy. Rev. Lett. 70, 818-821 (1993).

5. Raizen, M. G., Gilligan, J. M., Bergquist, J. C., Itano, W. M., and Wineland, D. J., Phys. Rev. A 45, 6493-6501 (1992).

6. Dubin, D. H. E., Phys. Rev. Lett. 71, 2753-2756 (1993).

\section{DISCLAIMER}

This report was prepared as an account of work sponsored by an agency of the United States Government. Neither the United States Government nor any agency thereof, nor any of their employees, makes any warranty, express or implied, or assumes any legal liability or responsibility for the accuracy, completeness, or usefulness of any information, apparatus, product, or process disclosed, or represents that its use would not infringe privately owned rights. Reference herein to any specific commercial product, process, or service by trade name, trademark, manufacturer, or otherwise does not necessarily constitute or imply its endorsement, recommendation, or favoring by the United States Government or any agency thereof. The views and opinions of authors expressed herein do not necessarily state or reflect those of the United States Government or any agency thereof. 